\title{
Tanshinone IIA induces autophagy in colon cancer cells through MEK/ERK/mTOR pathway
}

\author{
Jun Qian ${ }^{1 *}$, Yi Cao ${ }^{2 \#}$, Junfeng Zhang ${ }^{3}$, Lingchang $\mathrm{Li}^{4}$, Juan $\mathrm{Wu}^{5}$, Guoli $\mathrm{Wei}^{4}$, Jialin $\mathrm{Yu}^{4}$, Jiege $\mathrm{Huo}^{4}$ \\ ${ }^{1}$ Department of Diagnostics of Chinese Medicine, School of Chinese Medicine, School of Integrated Chinese and Western Medicine, Nanjing \\ University of Chinese Medicine, Nanjing, China; ${ }^{2}$ Research Office of Herbal Literature, Institute of Literature in Chinese Medicine, Nanjing \\ University of Chinese Medicine, Nanjing, China; ${ }^{3}$ Department of Pathogen and Immunology, School of Medicine \& Holistic Integrative Medicine, \\ Nanjing University of Chinese Medicine, Nanjing, China; ${ }^{4}$ Department of Oncology, Affiliated Hospital of Integrated Traditional Chinese and \\ Western Medicine, Nanjing University of Chinese Medicine, Nanjing, China; ${ }^{5}$ Department of Public health, School of Medicine \& Holistic \\ Integrative Medicine, Nanjing University of Chinese Medicine, Nanjing, China \\ Contributions: (I) Conception and design: J Qian, J Huo; (II) Administrative support: Y Cao; (III) Provision of study materials or patients: Y Cao, J \\ Zhang, G Wei; (IV) Collection and assembly of data: L Li, J Wu; (V) Data analysis and interpretation: J Qian; (VI) Manuscript writing: All authors; \\ (VII) Final approval of manuscript: All authors. \\ "These authors contributed equally to this work. \\ Correspondence to: Jun Qian. 138 Xianlin Rd, Nanjing, Jiangsu Province, 210023, PR China. Email: junqian_njucm@163.com; Jiege Huo, 100 Shizi \\ Street, Nanjing, Jiangsu Province, 210028, China. Email: huojiege@jsatcm.com.
}

Background: Colon cancer is a common malignancy of the digestive tract. The search for effective drugs to treat colon cancer has become the focus of current researches. Tanshinone IIA (Tan IIA) is a fat-soluble component extracted from tanshinone, a traditional Chinese medicine. Tan IIA can modulate the occurrence and development of tumors, but its effect on autophagy in colon cancer cells has not been reported.

Methods: Two types of colon cancer cell lines were selected and different concentrations of Tan IIA were used to treat cells at different time points. Cell Counting Kit-8 assay (CCK-8) was used to detect the effect of Tan IIA on cell proliferation; transmission electron microscopy was used to observe the formation of autophagosomes; reverse transcription-polymerase chain reaction (RT-qPCR) and western blot were used to detect the expression of autophagy related genes and proteins. Cell transfection was used to interfere with MEK (mitogen-activated extracellular signal-regulated kinase) expression, and RT-qPCR and western blot were used to detect the expression of MEK/ERK/mTOR pathway-related proteins.

Results: Tan IIA resulted in a significant reduction in the viability of the two kinds of colon cancer cells. The number of autophagosomes increased significantly after the treatment of Tan IIA into these cells. Addition of autophagy inhibitor 3-MA (3-Methyladenine) improved the increase of autophagosomes in cells induced by Tan IIA. At the same time, Tan IIA induced the expression of autophagy-related proteins in the two colon cancer cell lines. When Tan IIA induced autophagy in colon cancer cells, the expression of MEK/ ERK/mTOR pathway-related proteins increased significantly. After interfering with the expression of MEK, the expression of autophagy decreased significantly, indicating that Tan IIA promoted autophagy of colon cancer cells through MEK/ERK/mTOR pathway.

Conclusions: Tan IIA stimulates autophagy in colon cancer cells through MEK/ERK/mTOR pathway, hence inhibiting the growth of colon cancer cells.

Keywords: Tanshinone IIA; autophagy; colon cancer cells; MEK/ERK/mTOR pathway

Submitted Apr 30, 2020. Accepted for publication Sep 14, 2020.

doi: $10.21037 /$ tcr-20-1963

View this article at: http://dx.doi.org/10.21037/tcr-20-1963 


\section{Introduction}

Colon cancer is a common malignant tumor of the digestive tract, which usually occurs in the junction of rectum and sigmoid colon. The incidence of colon cancer is the highest in the age group of 40-50 years old, and the ratio of male to female with this disease is $2: 1$ (1). The incidence of gastrointestinal tumors ranks second among all carcinomas. In recent years, the incidence of colon cancer has become increasingly high. The existing treatment methods, such as surgery, chemotherapy, and radiotherapy, are limited in reducing mortality and improving prognosis, and the 5-year survival rate of patients is merely about $50 \%$. With changes of environmental factors and dietary habits, colon cancer has become a growing threat to human health (2). Thus, the search for effective drugs to treat colon cancer is the focus of this current research.

Tan IIA is a fat-soluble component extract, the main pharmacological effects of which are to improve coronary blood circulation (3), repair vascular endothelial cells (4), inhibit the formation of atherosclerosis (5), and prevent and treat the complications of diabetes (6). Recent studies have revealed that Tan IIA regulates the development of cancer by the regulation of proliferation, apoptosis and differentiation in cancerous cells. Tan IIA reverses EGF and TGF- $\beta 1$-mediated epithelial mesenchymal transformation in hepatoma carcinoma cells via the PI3K/Akt/ERK signaling pathway (7). Tan IIA reverses gefitinib resistance in human non-small cell lung cancer by modulating the VEGFR/Akt pathway (8). Moreover, there are several studies demonstrating the effect of Tan IIA on the proliferation, invasion, and apoptosis of colon cancer cells (9-11).

Autophagy is type II programmed cell death. As an intracellular decomposition process, autophagy is involved in energy metabolism, organelle transformation, growth regulation and senescence (12). It is characterized by the formation of autophagosomes and degradation of cellular components, such as mitochondria, ribosomes, and endoplasmic reticulum (13). A large number of studies have indicated that autophagy is closely connected to the development of tumors (14-16). Autophagy-induced cell death may provoke growth inhibition of tumor cells, thereby inhibiting tumor occurrence, suggesting that autophagy may be a potential breakthrough in the treatment of tumors. In addition, it has been reported that a variety of therapeutic drugs for tumors can regulate the proliferation, apoptosis, and differentiation of tumorous cells by acting on the autophagy. In colon cancer, Ophiopogon B induces autophagy and apoptosis of colon cancer cells through activating the $\mathrm{JNK} / \mathrm{c}$-jun signaling pathway to function as a tumor inhibitor (17). In xenograft tumor mice, curcumin inhibits tumor formation by suppressing AMPK/ULK1dependent autophagy and enhancing apoptosis of colon cancer cells (18). These results indicate that traditional Chinese medicine play an important role in the regulation of autophagy in the treatment of tumors. Whereas, the role of Tan IIA in autophagy of colon cancer cells has not been reported yet.

In this study, two colon cancer cell lines were selected to study the effect of Tan IIA on autophagy in colon cancer cells, and the specific mechanism has also been discussed, which may provide clinical evidence for the treatment of colon cancer by Tan IIA.

We present the following article in accordance with the MDAR checklist (available at http://dx.doi.org/10.21037/ tcr-20-1963).

\section{Methods}

\section{Cell culture and treatment}

Colon cancer cell lines SW480 (RRID:CVCL_0546) and HT29 (RRID:CVCL_0320) used in this study were obtained from Shanghai Cell Collection (Shanghai, China), and were cultured in DMEM (Gibco; Thermo Fisher Scientific) added with 10\% FBS (Gibco; Thermo Fisher Scientific) at $37{ }^{\circ} \mathrm{C}$ with $5 \% \mathrm{CO}_{2}$. Tan IIA was purchased from Selleckchem (Houston, TX, USA). Different concentrations of Tan IIA were used to induce colon cancer cell lines for 24, 48, 72 and 96 h. The Control group was given the same dose of DMEM for the same duration of induction.

\section{Cell Counting Kit-8 (CCK-8) assay}

Cells were seeded into 96 -well plates and cultivated at $37^{\circ} \mathrm{C}$ in a humidified incubator with $5 \% \mathrm{CO}_{2}$. Cell viability was determined using the CCK-8 reagent (Dojindo Molecular Technologies, Inc.), according to the manufacturer's protocol. Different concentrations of Tan IIA were used to induce colon cancer cell lines for 24, 48, 72 and $96 \mathrm{~h}$. After treatment of cells, $10 \mu \mathrm{L}$ CCK-8 solution (Dojindo Molecular Technologies, Inc.) was added to each well for nearly $4 \mathrm{~h}$, and then the optical density was measured at $450 \mathrm{~nm}$ using a microplate reader. 


\section{Reverse transcription-polymerase chain reaction (RT-qPCR)}

Total RNA was isolated from cells using TRIzol reagent (Invitrogen; Thermo Fisher Scientific, Inc.). RNA (2 $\mu \mathrm{g})$ was reverse transcribed into cDNA using the iQ5 Multicolor Real-Time PCR Detection system (Bio-Rad Laboratories, Inc.). Subsequently, Real-time PCR analysis was carried out via iQ5 Multicolor Real-Time PCR Detection system (BIO-RAD, USA). And the DNA bands were standardized to that of GAPDH. The primer pairs were as follows: LC3 forward, 5'-GAACGATACAAGGGTGAGAAGCA-3' and reverse, 5'-TGAGATTGGTGTGGAGACGCT-3'; p62 forward, 5'-TGAGATTGGTGTGGAGACGCT-3' and reverse, 5'-ACAGCATCTGGGAGAGGGACT-3'; Beclin1 forward, 5'-ATCTGGCACAGTGGACAGTTTG-3' and reverse, 5'-CCGTAAGGAACAAGTCGGTATC-3'; ATG7 forward, 5'-CAGAAAGGAGGCATGGGACC-3' and reverse, 5'-AGACACAACCTTGTCCAAGT-3'; FOXO3 forward, 5'-GGACCTGGACATGTTCAATG-3' and reverse, 5'-CCTGCTTAGCACCAGTGAAG-3'; GAPDH forward, 5'-CCTCTGACTTCAACAGCGACAC-3'; and reverse, 5'-CTGTTGCTGTAGCCAAATTCGT-3'.

\section{Transmission electron microscopy}

After treatment, cells were washed with PBS, collected, centrifuged at $800 \mathrm{r} / \mathrm{min}$ for $8 \mathrm{~min}$, and then made into clusters. Next, cells were fixed with $2.5 \%$ electron microscope solution glutaraldehyde overnight at $4{ }^{\circ} \mathrm{C}$ and rinsed with PBS. The solution was fixed utilizing $1 \%$ osmic acid at $4{ }^{\circ} \mathrm{C}$ and washed again with PBS. After that, the cells were dehydrated, followed by solidification of cells through embedding in the resin. The fixed wax blocks were sectioned by a supermodel slicer, double-stained with $3 \%$ uranium acetate and lead citrate, and observed and photographed under a transmission electron microscope (Hitachi H-7650).

\section{Western blot}

The treated cells were collected and lysed with RIPA lysis buffer (Beyotime Institute of Biotechnology) and incubated for $30 \mathrm{~min}$ on ice. Then proteins were detected using a BCA protein assay kit (Bio-Rad Laboratories, Inc.). A total of $40 \mu \mathrm{g}$ protein was loaded onto $10 \%$ SDS-polyacrylamide gels to separate various proteins, which were subsequently transferred to PVDF membranes. The membranes were blocked with $10 \%$ skim milk for $2 \mathrm{~h}$ at room temperature, followed by incubation with primary antibodies for one night at $4{ }^{\circ} \mathrm{C}$. Subsequently, the membranes were incubated with goat anti-rabbit horseradish peroxidase-conjugated IgG secondary antibodies (1:5,000; AA24142) at room temperature for just $1 \mathrm{~h}$. The signals were detected using enhanced chemiluminescence reagent (GE Healthcare) and Image J software (version 146; National Institutes of Health, Bethesda) was used to analyze fold-changes of protein levels. Anti-Beclin 1 (1:1,000; E2200675), anti-ATG7 (1:1,000; E90690), anti-FOXO3 (1:1,000; E0220202), anti-p62 (1:1,000; E2510713), anti- $\beta$-catenin (1:1,000; 13 8400, RRID:AB_2533039), anti-Cyclin-D1 (1:1,000; MA516356, RRID:AB_2537875), anti- $\beta$-actin (1:1,000; E81102-1) antibodies were obtained from EnoGene, Inc.

\section{Plasmid construction and shRNA interference assay}

A short hairpin RNA (shRNA) sequence targeting MEK was obtained from Genechem Company (Shanghai, China). A scrambled shRNA was included as a negative control.

\section{Statistical analysis}

Data obtained from three independent groups were expressed as the mean \pm standard deviation. SPSS 17.0 statistical software (SPSS, Inc., Chicago, IL, USA, RRID:SCR_002865) was used for all statistical analyses. Comparisons between groups were analyzed by Student's $t$-test or one-way analysis of variance followed by Tukey's test. $\mathrm{P}<0.05$ was considered to indicate a statistically significant difference.

\section{Results}

\section{Tan IIA inbibited the proliferation of colon cancer cells}

CCK-8 was used to detect the proliferation of colon cancer cells (SW480 and HT29) treated with Tan IIA at different concentrations. We found the decline in cell viability with the increases in concentration and treatment time of Tan IIA, indicating that Tan IIA could inhibit the proliferation of colon cancer cells (Figure 1A,B). Combined with the previous literature, we chose $10 \mu M$ Tan IIA for the following experiments.

\section{Tan IIA promoted autophagy of colon cancer cells}

In our paper, we induced SW480 and HT29 cells for 24, 

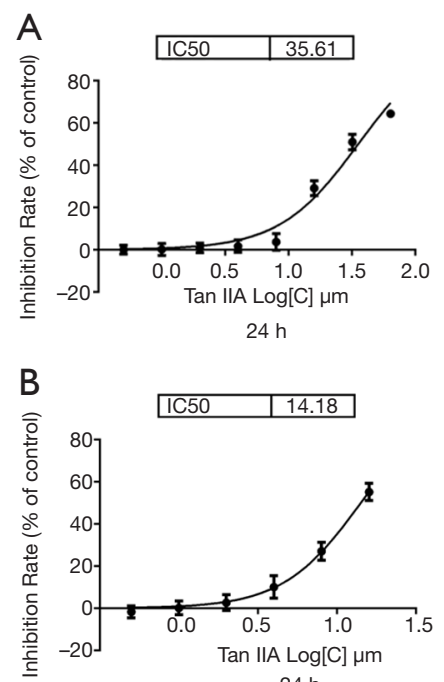

$24 \mathrm{~h}$

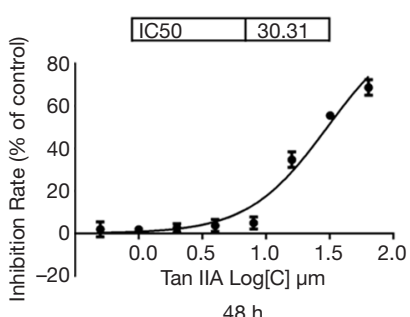

$48 \mathrm{~h}$

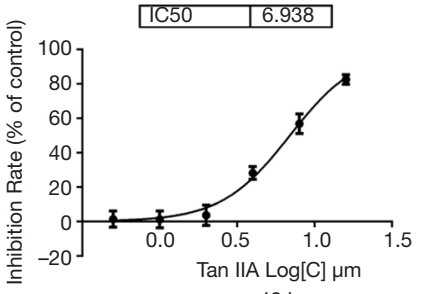

$48 \mathrm{~h}$

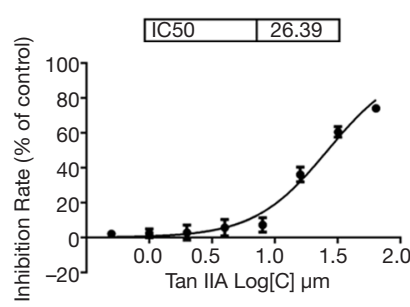

$72 \mathrm{~h}$

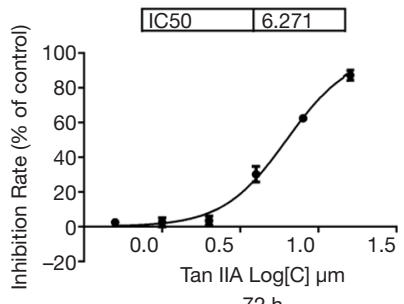

$72 \mathrm{~h}$

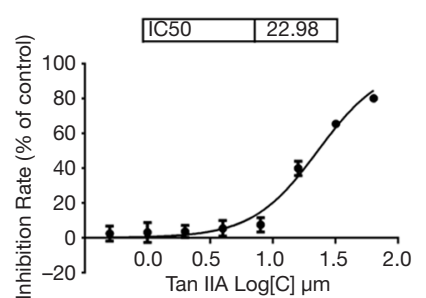

$96 \mathrm{~h}$

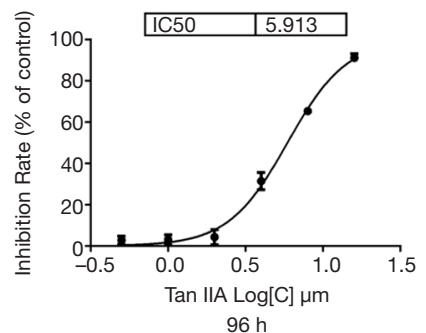

$96 \mathrm{~h}$

Figure 1 Selection of Tan IIA concentration. IC50 of Tan IIA was determined by CCK-8.

Control

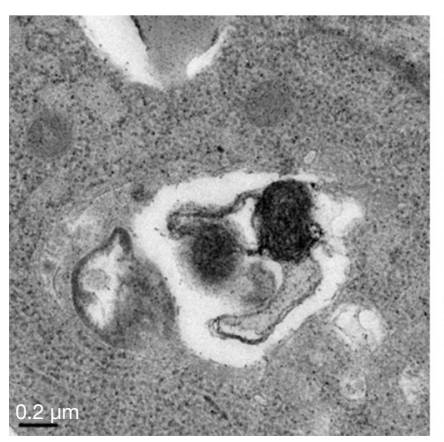

Tan IIA

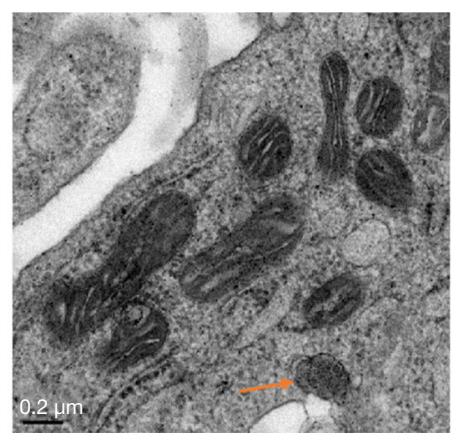

3-MA

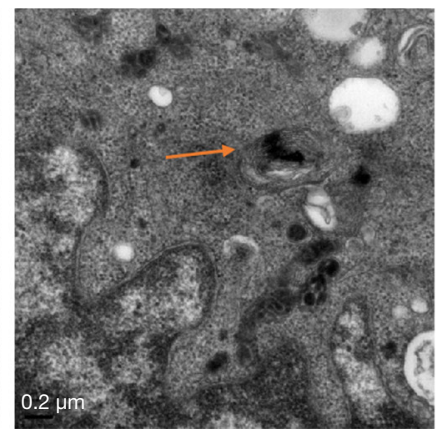

Tan IIA+3-MA

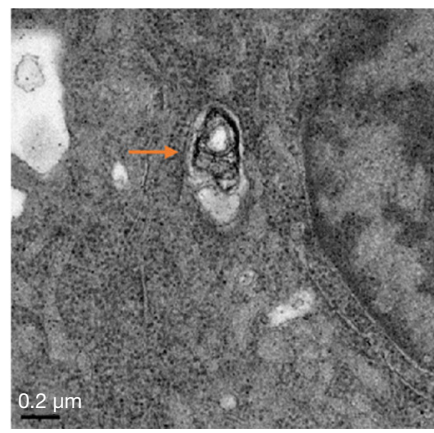

Figure 2 Effect of Tan IIA on autophagosome. The level of autophagosome was observed under electron microscope. Magnification $\times 4,000$.

The arrows show the autophagosomes.

48 and $72 \mathrm{~h}$ with $10 \mu \mathrm{M}$ Tan IIA, and explored the effect of Tan IIA on the autophagy of colon cancer cell lines by adding autophagy inhibitor 3-MA. The cells were divided into four groups: control, Tan IIA, 3-MA, and TanIIA+3-MA. The results presented that the endoplasmic reticulum of the control group was normal with a few autophagosomes. The endoplasmic reticulum of the Tan IIA group expanded under stress, and there were a large number of autophagosomes. In the 3-MA group, the shape of mitochondria was changed, while the endoplasmic reticulum was not significantly changed. Also, there was slight autophagy. In the TanIIA+3-MA group, endoplasmic reticulum stress and some autophagosomes were observed. The results suggested that compared with the control group, the cells in the Tan IIA group showed obvious autophagy induction. With the addition of autophagy inhibitor 3-MA, the induction of autophagy by Tan IIA was reduced (Figure 2). Then, the expression of autophagyrelated proteins LC3, P62, Beclin1, ATG7 and FOXO3 was detected by RT-qPCR and western blot. Figure $3 A$ is the experimental result of RT-qPCR on SW480 cells, Figure $3 B$ is the experimental result of western blot on SW480 cells. Figure $4 A$ is the experimental result of RT-qPCR on HT29 cells, Figure $4 B$ is the experimental result of western blot on HT-29 cells. And results from which were consistent with the electron microscope results, indicating that Tan IIA could induce autophagy in colon cancer cells.

\section{Tan IIA activated autophagy through the activation of MEK/ERK/mTOR signaling patbway in colon cancer cells}

We found that when Tan IIA induced autophagy in cells, 
A
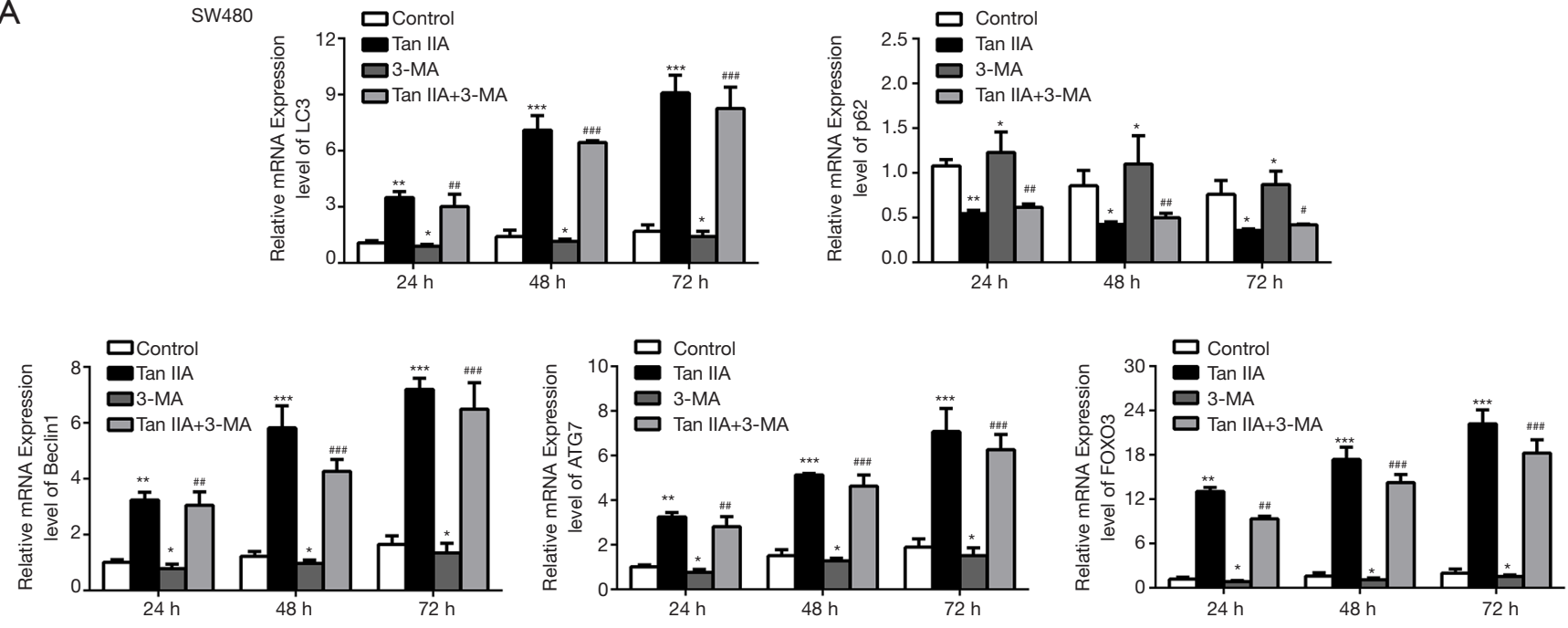

B

SW480-24 h
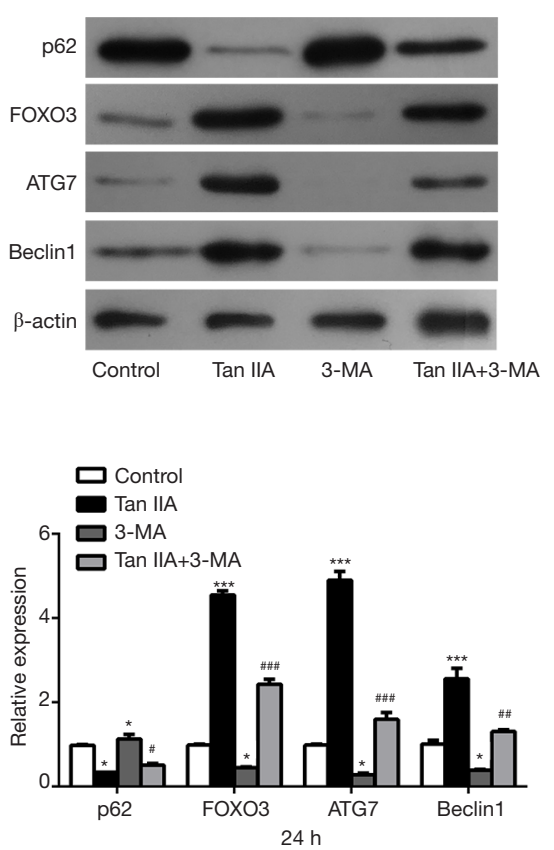

SW480-48 h
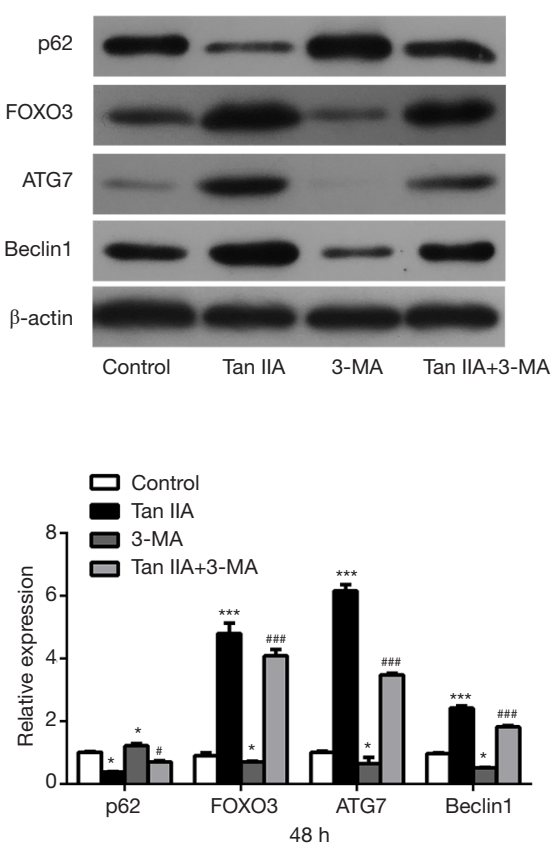

SW480-72 h
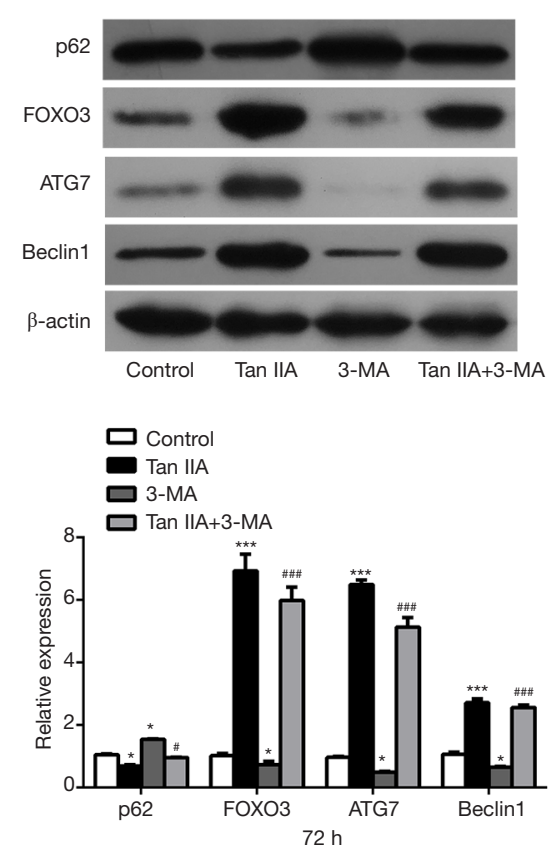

Figure 3 Effect of Tan IIA on autophagy in SW480 cells. (A) RT-qPCR was used to detect the expression of autophagy-related genes LC3, P62, Beclin1, ATG7 and FOXO3. (B) Western blot was utilized to analyze the expression of autophagy-related proteins LC3, P62, Beclin1, ATG7 and FOXO3. * $, \mathrm{P}<0.05,{ }^{* *}, \mathrm{P}<0.01,{ }^{* * *}, \mathrm{P}<0.001$ vs. control; ${ }^{*}, \mathrm{P}<0.05,{ }^{* \#}, \mathrm{P}<0.01,{ }^{\# \# \#}, \mathrm{P}<0.001$ vs. 3-MA. Tan IIA, tanshinone IIA; 3-MA, 3-Methyladenine; FOXO3, Forkhead box O3.

MEK/ERK/mTOR signaling pathway was activated. Compared with the control group, the expression of MEK, ERK, and mTOR increased significantly after Tan IIA was given but decreased significantly in the 3-MA group. Compared with the 3-MA group, the protein expressions of MEK, ERK, and mTOR in the Tan IIA+3-MA group were increased, hinting that Tan IIA reversed the inhibitory effect of 3-MA on autophagy in colon cancer cells (Figure $5 A, B$ ). The results showed that Tan IIA may regulate the autophagy of colon cancer cells through MEK/ ERK/mTOR Pathway. To further verify our conclusion, we interfered with MEK expression through cell transfection 
A

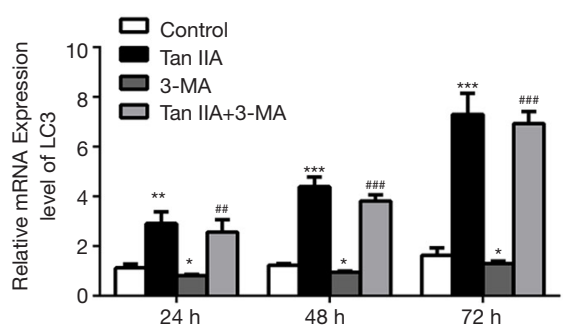

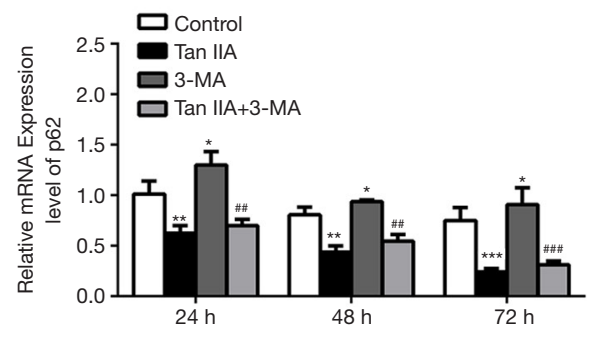
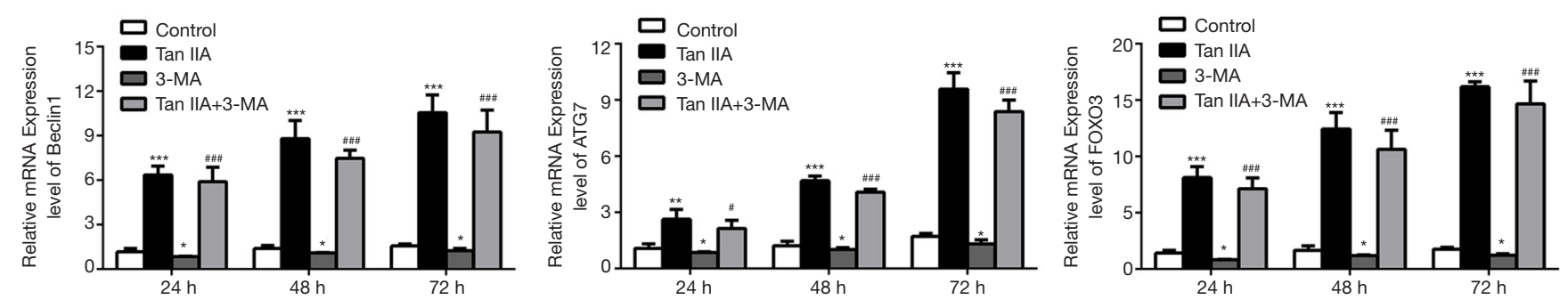

B
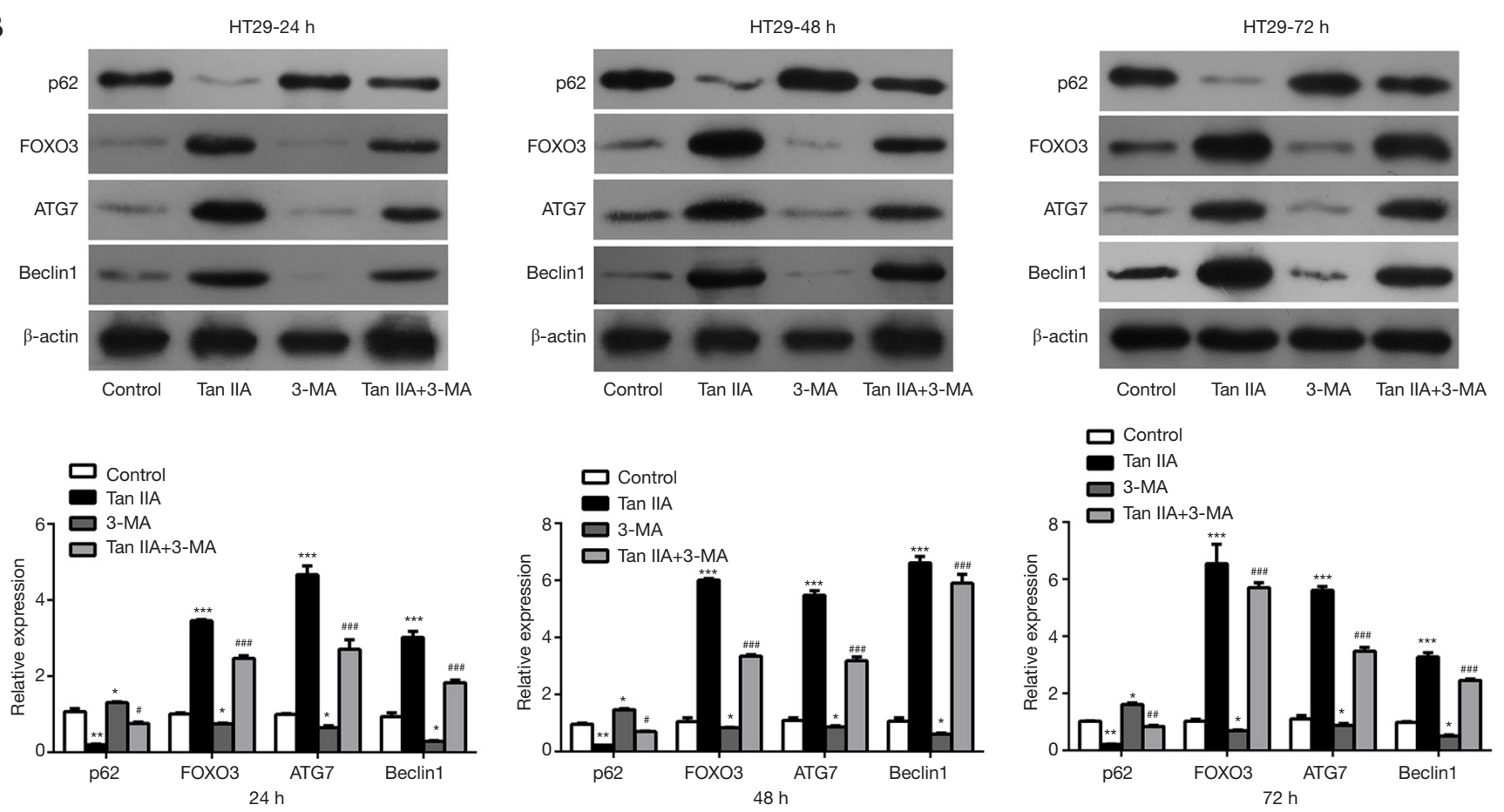

Figure 4 Effect of Tan IIA on autophagy level in HT29 cells. (A) RT-qPCR dissected the expression of autophagy-associated genes LC3, P62, Beclin1, ATG7 and FOXO3. (B) Western blot was applied to detect the expression of autophagy-associated proteins LC3, P62, Beclin1, ATG7 and FOXO3. ${ }^{* *}, \mathrm{P}<0.01,{ }^{* * *}, \mathrm{P}<0.001$ vs. control; ${ }^{*}, \mathrm{P}<0.05,{ }^{, \#}, \mathrm{P}<0.01,{ }^{\# \# \#}, \mathrm{P}<0.001$ vs. 3-MA. Tan IIA, tanshinone IIA; 3-MA, 3-Methyladenine; FOXO3, Forkhead box O3.

technology (Figure 6A), and divided the cells into control, shRNA-NC, shRNA-MEK, shRNA-NC+TanIIA, and shRNA-MEK+TanIIA groups. As shown in Figure 6B, western blot was used to detect the expression of pathwayand autophagy-related proteins. It was viewed that after the inhibition of MEK, the expression of pathway- related proteins MEK, ERK, and mTOR was decreased, the expression of autophagy protein ATG7 and Beclin1 decreased, while the expression of P62 was increased. Compared with the shRNA-MEK group, the expression of MEK, ERK, mTOR, ATG7 and Beclin1 was raised and the expression of P62 was reduced in the shRNA-MEK+TanIIA 
A

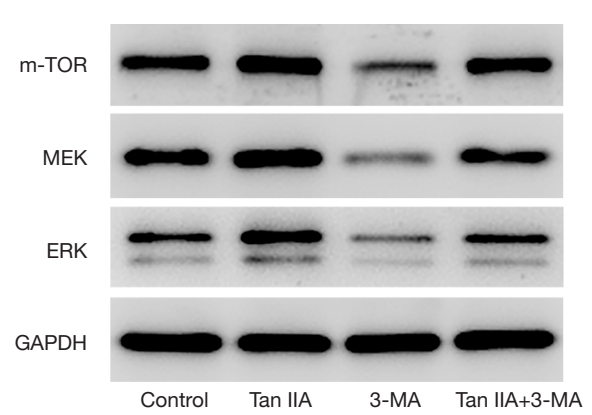

B

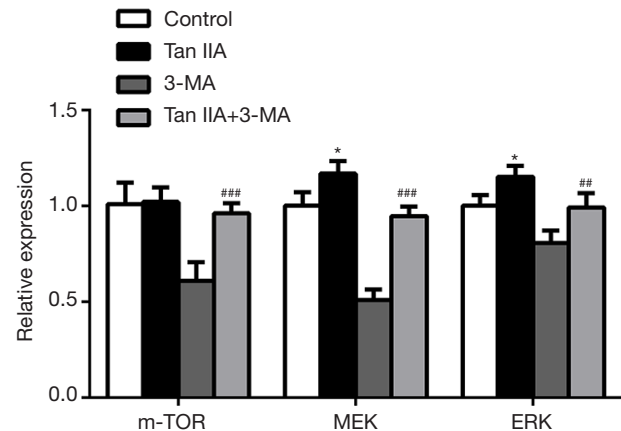

Figure 5 Effect of Tan IIA on the MEK/ERK/mTOR pathway. (A) The expression of MEK, ERK and mTOR was detected by western blot. (B) The statistical analysis chart of A. *, $\mathrm{P}<0.05$ vs. control; ${ }^{\#}, \mathrm{P}<0.01$, ${ }^{\# \#}, \mathrm{P}<0.001$ vs. 3-MA. Tan IIA, tanshinone IIA; 3-MA, 3-Methyladenine; FOXO3, Forkhead box O3; MER, mitogen-activated extracellular signal-regulated kinase; ERK, extracellular regulated protein kinases; mTOR, mammalian target of rapamycin.

A

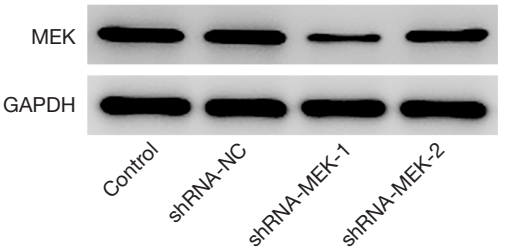

C

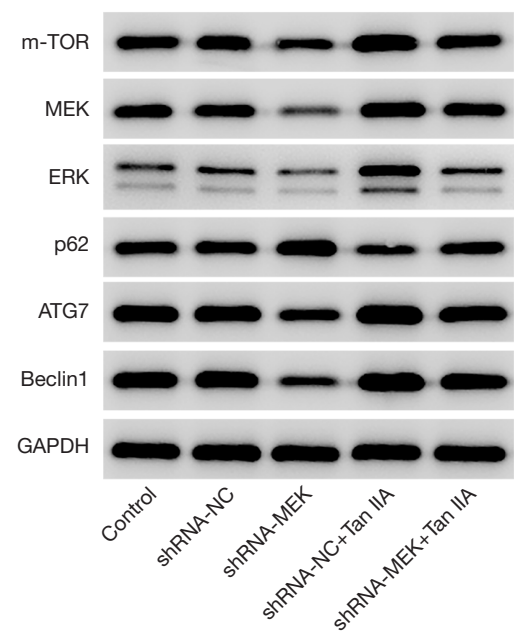

B

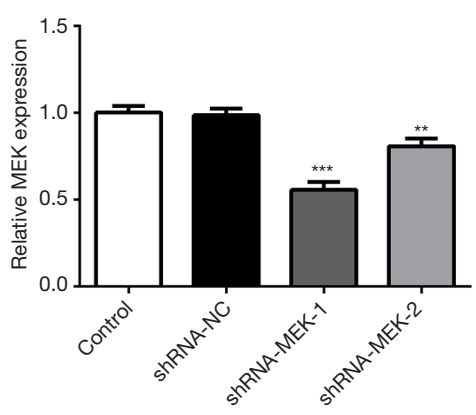

D

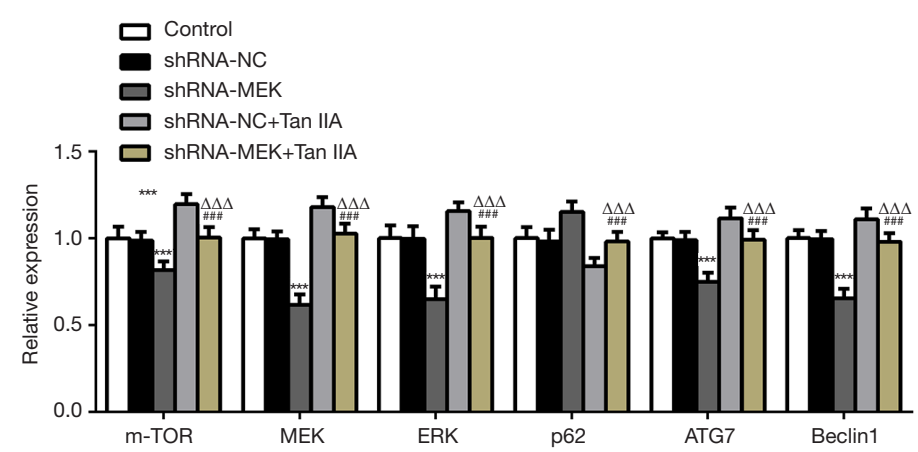

Figure 6 Tan IIA stimulated autophagy in colon cancer cells by activating the MEK/ERK/mTOR pathway. (A) The expression of MEK protein after transfection was detected by western blot. (B) The statistical analysis chart of A. ${ }^{* *}, \mathrm{P}<0.01,{ }^{* * *}, \mathrm{P}<0.001 v s$. shRNA-NC. (C) The expression levels of pathway-related molecules MEK, ERK and mTOR, and those of autophagy-related proteins p62, ATG7 and

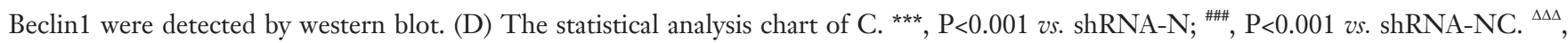
$\mathrm{P}<0.001$ vs. shRNA-NC+Tan IIA. Tan IIA, tanshinone IIA; 3-MA, 3-Methyladenine; FOXO3, Forkhead box O3; MER, mitogen-activated extracellular signal-regulated kinase; ERK, extracellular regulated protein kinases; mTOR, mammalian target of rapamycin. 
group. Compared with shRNA-NC+Tan IIA group, the expression of MEK, ERK, mTOR, ATG7, and Beclin1 was lessened but that of P62 protein was elevated in shRNAMEK+Tan IIA group. These experimental results exhibited that Tan IIA activated autophagy through promotion of MEK/ERK/mTOR signaling pathway in colon cancer cells.

\section{Discussion}

Tan IIA is a monomer compound isolated from tanshinone, which has a wide range of pharmacological functions including anti-oxidation (19), anti-atherosclerosis (5), anti-tumor (20), inhibition of thrombosis and inhibition of platelet aggregation (21). Currently, Tan IIA is mainly used in the treatment of cardiovascular diseases. However, more and more studies have proved that Tan IIA plays a considerable role in the development of tumors $(8,22,23)$. Tan IIA can inhibit the proliferation, invasion and migration of colon cancer cells, and promote the apoptosis of colon cancer cells. In addition, emerging study has illustrated that Tan IIA can inhibit the formation of microvascular of tumor tissues by reducing the expression level of serum VEGF and cause a dose-dependent inhibition of colon cancer tumor growth (24). Therefore, in this paper, we used Tan IIA with different concentrations to treat colorectal cancer cell lines at different time points, and the results from CCK-8 displayed that with the increase in time and concentration of Tan IIA treatment, the cell activity decreased markedly.

Autophagy is associated with cell death. Moderate degree of autophagy can promote cell survival, but excessive autophagy can accelerate cell death, thus maintaining the physiological balance within the cells (25). Autophagy has been a research hotspot in the field of life science since its discovery. Commonly, it regulates intracellular repair and damage through multiple signaling pathways, thus exerting effects in the occurrence and development of carcinomas (26). Previous papers have affirmed that Tan IIA has a vital role in the proliferation, invasion, migration and apoptosis of colon cancer cells, however, the role of Tan IIA in the autophagy of colon cancer cells has not been investigated in detail. Nowadays, there are many reports on the role of TCM in regulating autophagy in colon cancer cells, In colon cancer, Ophiopogon B induces autophagy and apoptosis of colon cancer cells through activating the $\mathrm{JNK} / \mathrm{c}$-jun signaling pathway to function as an tumor inhibitor (17). In xenograft tumor mice, curcumin inhibits tumor formation by suppressing AMPK/ULK1-dependent autophagy and enhancing apoptosis of colon cancer cells (18). Therefore, in our paper, we detected the expression of autophagy-related indicators. We discovered that the level of autophagy increased after the addition of Tan IIA to SW480 and HT29 cells, which was reversed by co-treatment of autophagy inhibitor 3-MA, indicating that Tan IIA could promote autophagy. The MEK/ERK pathway, an autophagy signaling pathway, can induce autophagy and cell death (27). In the human cervical cancer cells, Concanavalin A (Con A) activates autophagy by up-regulating the MEK/ERK/mTOR signaling pathway and participates in cell apoptosis, thus hampering the growth and proliferation (28). 8-CEPQ from onion beef soup can repress colon cancer through ERK signaling pathway and accelerate autophagy death of colon cancer cells (29). In addition, Wang et al. (30) reported that Tan IIA activates autophagy via the MEK/ERK/mTOR pathway to reduce hepatic ischemia-reperfusion injury. mTOR protein, an atypical serine/threonine kinase, is the main regulator of autophagy, which can regulate the metabolism of cells and proteins in the body, and is widely involved in the occurrence and development of tumors (31). Study has shown that the consumption of autophagy inhibitor $\mathrm{uL} 3$ in colon cancer cells can increase the drug resistance of colon cancer cells by inducing autophagy, and the recovery of uL3 can drive the apoptosis of colon cancer cells by inhibiting autophagy (32). Therefore, we speculated that Tan IIA could boost autophagy of colon cancer cells SW480 and HT29 through activation of the MEK/ERK/mTOR signaling pathway, thus induce apoptosis of cells. It was observed in this work that $\mathrm{MEK} / \mathrm{ERK} / \mathrm{mTOR}$ signaling pathway was activated after the induction of autophagy by Tan IIA. Next, we interfered with MEK expression and further disclosed that Tan IIA induced autophagy through the activation of MEK/ERK/mTOR signaling pathway in colon cancer cells. However, we had no idea whether the significantly decreased cell viability after Tan IIA addition was related to autophagy and autophagic death in colon cancer cells. This remains to be further studied in our future research.

\section{Conclusions}

Tan IIA induces autophagy in colon cancer cells through MEK/ERK/mTOR pathway. This article provides more experimental basis for Tan IIA in the clinical treatment of colon cancer. 


\section{Acknowledgments}

Funding: This work was supported by Project of National Clinical Research Base of Traditional Chinese Medicine in Jiangsu Province, China (No. JD2019SZXYB04); a Project Funded by the Priority Academic Program Development of Jiangsu Higher Education Institutions (Integration of Chinese and Western Medicine); the Leading Talents Project of Chinese Medicine in Jiangsu Province (No. SLJ0211).

\section{Footnote}

Reporting Checklist: The authors have completed the MDAR checklist. Available at http://dx.doi.org/10.21037/tcr-201963

Data Sharing Statement: Available at http://dx.doi. org/10.21037/tcr-20-1963

Conflicts of Interest: All authors have completed the ICMJE uniform disclosure form (available at http://dx.doi. org/10.21037/tcr-20-1963). The authors have no conflicts of interest to declare.

Ethical Statement: The authors are accountable for all aspects of the work in ensuring that questions related to the accuracy or integrity of any part of the work are appropriately investigated and resolved. This experiment does not involve animal or human experiments.

Open Access Statement: This is an Open Access article distributed in accordance with the Creative Commons Attribution-NonCommercial-NoDerivs 4.0 International License (CC BY-NC-ND 4.0), which permits the noncommercial replication and distribution of the article with the strict proviso that no changes or edits are made and the original work is properly cited (including links to both the formal publication through the relevant DOI and the license). See: https://creativecommons.org/licenses/by-nc-nd/4.0/.

\section{References}

1. Siegel RL, Miller KD, Fedewa SA, et al. Colorectal cancer statistics, 2017. CA Cancer J Clin 2017;67:177-93.

2. Benson AB, Venook AP, Al-Hawary MM, et al. NCCN Guidelines Insights: Colon Cancer, Version 2.2018. J Natl Compr Canc Netw 2018;16:359-69.
3. Long R, You Y, Li W, et al. Sodium tanshinone IIA sulfonate ameliorates experimental coronary no-reflow phenomenon through down-regulation of FGL2. Life Sci 2015;142:8-18.

4. Li Z, Zhang S, Cao L, et al. Tanshinone IIA and Astragaloside IV promote the angiogenesis of mesenchymal stem cell-derived endothelial cell-like cells via upregulation of $\mathrm{Cx} 37, \mathrm{Cx} 40$ and $\mathrm{Cx} 43$. Exp Ther Med 2018;15:1847-54.

5. Zhu J, Xu Y, Ren G, et al. Tanshinone IIA Sodium sulfonate regulates antioxidant system, inflammation, and endothelial dysfunction in atherosclerosis by downregulation of CLIC1. Eur J Pharmacol 2017;815:427-36.

6. Ri-Ge-le A, Guo ZL, Wang Q, et al. Tanshinone IIA Improves Painful Diabetic Neuropathy by Suppressing the Expression and Activity of Voltage-Gated Sodium Channel in Rat Dorsal Root Ganglia. Exp Clin Endocrinol Diabetes 2018;126:632-9.

7. Zhang $\mathrm{L}$, Lin $\mathrm{W}$, Chen $\mathrm{X}$, et al. Tanshinone IIA reverses EGF- and TGF-beta1-mediated epithelial-mesenchymal transition in HepG2 cells via the PI3K/Akt/ERK signaling pathway. Oncol Lett 2019;18:6554-62.

8. Wang R, Luo Z, Zhang H, et al. Tanshinone IIA Reverses Gefitinib-Resistance In Human Non-Small-Cell Lung Cancer Via Regulation Of VEGFR/Akt Pathway. Onco Targets Ther 2019;12:9355-65.

9. Xue J, Jin X, Wan X, et al. Effects and Mechanism of Tanshinone II A in Proliferation, Apoptosis, and Migration of Human Colon Cancer Cells. Med Sci Monit 2019;25:4793-800.

10. Ma S, Lei Y, Zhang L, et al. Research on the inhibiting effect of tanshinone IIA on colon cancer cell growth via COX-2-Wnt/beta-catenin signaling pathway. J BUON 2018;23:1337-42.

11. Shan YF, Shen X, Xie YK, et al. Inhibitory effects of tanshinone II-A on invasion and metastasis of human colon carcinoma cells. Acta Pharmacol Sin 2009;30:1537-42.

12. Ravanan P, Srikumar IF, Talwar P. Autophagy: The spotlight for cellular stress responses. Life Sci 2017;188:53-67.

13. Lamark T, Svenning S, Johansen T. Regulation of selective autophagy: the p62/SQSTM1 paradigm. Essays Biochem 2017;61:609-24.

14. Yang A, Herter-Sprie G, Zhang H, et al. Autophagy Sustains Pancreatic Cancer Growth through Both CellAutonomous and Nonautonomous Mechanisms. Cancer Discov 2018;8:276-87. 
15. Krishan S, Sahni S, Leck LYW, et al. Regulation of autophagy and apoptosis by Dp44mT-mediated activation of AMPK in pancreatic cancer cells. Biochim Biophys Acta Mol Basis Dis 2020:165657.

16. Li JP, Zhang HM, Liu MJ, et al. miR-133a-3p/FOXP3 axis regulates cell proliferation and autophagy in gastric cancer. J Cell Biochem 2020;121:3392-405.

17. Gao GY, Ma J, Lu P, et al. Ophiopogonin B induces the autophagy and apoptosis of colon cancer cells by activating JNK/c-Jun signaling pathway. Biomed Pharmacother 2018;108:1208-15.

18. Zhang P, Lai ZL, Chen HF, et al. Curcumin synergizes with 5-fluorouracil by impairing AMPK/ULK1-dependent autophagy, AKT activity and enhancing apoptosis in colon cancer cells with tumor growth inhibition in xenograft mice. J Exp Clin Cancer Res 2017;36:190.

19. Lin TJ. Antioxidation mechanism of schizandrin and tanshinonatic acid $\mathrm{A}$ and their effects on the protection of cardiotoxic action of adriamycin. Sheng Li Ke Xue Jin Zhan 1991;22:342-5.

20. Li Y, Jiang B, Wang R, et al. Synergistic effects of tanshinone IIA and andrographolide on the apoptosis of cancer cells via crosstalk between p53 and reactive oxygen species pathways. Pharmacol Rep 2020;72:400-17.

21. Wang H, Zhong L, Mi S, et al. Tanshinone IIA prevents platelet activation and down-regulates CD36 and MKK4/ JNK2 signaling pathway. BMC Cardiovasc Disord 2020;20:81.

22. Ma L, Jiang H, Xu X, et al. Tanshinone IIA mediates SMAD7-YAP interaction to inhibit liver cancer growth by inactivating the transforming growth factor beta signaling pathway. Aging (Albany NY) 2019;11:9719-37.

23. Wang M, Zeng X, Li S, et al. A Novel Tanshinone Analog Exerts Anti-Cancer Effects in Prostate Cancer by Inducing
Cell Apoptosis, Arresting Cell Cycle at G2 Phase and Blocking Metastatic Ability. Int J Mol Sci 2019;20:4459.

24. Zhou LH, Hu Q, Sui H, et al. Tanshinone II--a inhibits angiogenesis through down regulation of COX-2 in human colorectal cancer. Asian Pac J Cancer Prev 2012;13:4453-8.

25. Liang X, Liu L, Wang Y, et al. Autophagy-driven NETosis is a double-edged sword - Review. Biomed Pharmacother 2020;126:110065.

26. Hegedus C, Boros G, Fidrus E, et al. PARP1 Inhibition Augments UVB-Mediated Mitochondrial ChangesImplications for UV-Induced DNA Repair and Photocarcinogenesis. Cancers (Basel) 2019;12:5.

27. Wang J, Whiteman MW, Lian H, et al. A non-canonical MEK/ERK signaling pathway regulates autophagy via regulating Beclin 1. J Biol Chem 2009;284:21412-24.

28. Roy B, Pattanaik AK, Das J, et al. Role of PI3K/Akt/ mTOR and MEK/ERK pathway in Concanavalin A induced autophagy in HeLa cells. Chem Biol Interact 2014;210:96-102.

29. Zhao Y, Fan D, Zheng ZP, et al. 8-C-(E-phenylethenyl) quercetin from onion/beef soup induces autophagic cell death in colon cancer cells through ERK activation. Mol Nutr Food Res 2017;61. doi: 10.1002/mnfr.201600437.

30. Wang Y, Ni Q, Ye Q, et al. Tanshinone IIA activates autophagy to reduce liver ischemia-reperfusion injury by MEK/ERK/mTOR pathway. Pharmazie 2018;73:396-401.

31. Gogiraju R, Hubert A, Fahrer J, et al. Endothelial Leptin Receptor Deletion Promotes Cardiac Autophagy and Angiogenesis Following Pressure Overload by Suppressing Akt/mTOR Signaling. Circ Heart Fail 2019;12:e005622.

32. Pecoraro A, Carotenuto P, Franco B, et al. Role of uL3 in the Crosstalk between Nucleolar Stress and Autophagy in Colon Cancer Cells. Int J Mol Sci 2020;21:2143.
Cite this article as: Qian J, Cao Y, Zhang J, Li L, Wu J, Wei G, Yu J, Huo J. Tanshinone IIA induces autophagy in colon cancer cells through MEK/ERK/mTOR pathway. Transl Cancer Res 2020;9(11):6919-6928. doi: 10.21037/tcr-20-1963 